Bull. Austral. Math. Soc.

57R30

Vol. 46 (1992) [55-58]

\title{
ON THE SIGNATURE OF GENERALISED SEIFERT FIBRATIONS
}

\author{
Peter Y. Pang
}

In this note, we prove a signature product formula for generalised Seifert fibrations. We also discuss how this result can be viewed using the theory of minimal models.

\section{INTRODUCTION}

In this short note, we deduce a signature product formula for generalised Seifert fibrations based on results of Haefliger [6] and Chern-Hirzebruch-Serre [1]. Then we note that Haefliger's result has a natural interpretation from the point of view of the theory of minimal models, and the signature formula obtained can be viewed as a generalisation of Chern-Hirzebruch-Serre. Further, we demonstrate that the well-known result: that a compact, connected, orientable Riemannian manifold with a non-singular Killing vector-field has signature zero, can be interpreted in the same vein.

\section{Generalised Seifert Fibrations}

Definition 2.1: A foliation $\mathcal{F}$ is called a generalised Seifert fibration if (i) all its leaves are compact, and (ii) it is locally stable; equivalently, each leaf has a finite holonomy group, and $\mathcal{F}$ is Riemannian and taut [8]. See for example [2].

REMARK 2.2: It follows that the leaf space $M / \mathcal{F}$ of a generalised Seifert fibration on a manifold $M$ is a Satake manifold, and its cohomology (as a Satake manifold) coincides with the cohomology of the complex of basic differential forms of the foliation, $H_{B}(\mathcal{F})$. Also, the leaves of $\mathcal{F}$ have a comon holonomy covering, known as the universal leaf, which is a compact, connected manifold.

Theorem 2.3. Let $\mathcal{F}$ be a generalised Seifert fibration on a connected, compact, simply-connected manifold $M$. Let $\mathcal{L}$ denote the universal leaf of $\mathcal{F}$. Then, there is a signature product formula as follows:

$$
\operatorname{sign}(M)=\operatorname{sign}(\mathcal{L}) \cdot \operatorname{sign}\left(H_{B}(\mathcal{F})\right)
$$

Proof: By [6], there is a locally trivial fibration

$$
\mathcal{L} \longrightarrow M \longrightarrow B \Gamma
$$

Received 10 December 1991

Copyright Clearance Centre, Inc. Serial-fee code: 0004-9729/92 SA2.00+0.00. 
where $B \Gamma$ is the classifying space of the transverse holonomy groupoid of $\mathcal{F}$. Furthermore, there is a map

$$
\boldsymbol{B} \Gamma \longrightarrow M / \mathcal{F}
$$

which induces an isomorphism in rational cohomology. Thus, we have

$$
H^{\cdot}(B \Gamma) \cong H^{\cdot}(M / \mathcal{F}) \cong H_{B} \cdot(\mathcal{F})
$$

By the duality theorem of Kamber-Tondeur $[8], H_{B}(\mathcal{F})$ satisfies Poincaré duality, hence by [1], we have

$$
\begin{aligned}
\operatorname{sign}(M) & =\operatorname{sign}(\mathcal{L}) \cdot \operatorname{sign}(B \Gamma) \\
& =\operatorname{sign}(\mathcal{L}) \cdot \operatorname{sign}\left(H_{B}(\mathcal{F})\right)
\end{aligned}
$$

Corollary 2.4. Let $M$ be a connected, compact, simply-connected manifold with non-zero signature. Let $\mathcal{F}$ be a Riemannian foliation on $M$. Then the codimension of $\mathcal{F}$ is divisible by 4 .

Proof: By a theorem of Ghys [3], $\mathcal{F}$ can be approximated by a generalised Seifert fibration of the same codimension. Thus the corollary follows from the above theorem.

\section{Minimal models}

Definition 3.1: Let $\mathcal{F}$ be a foliation on a connected manifold $M$. Then, by the minimal model ( $\Lambda$-minimal $\Lambda$-extension) of $\mathcal{F}$, we mean the minimal model ( $\Lambda$-minimal $\Lambda$-extension) of the differential graded algebra map given by the inclusion of the basic differential forms into the de Rham algebra of $M, \Omega_{B}(\mathcal{F}) \longrightarrow \Omega_{D R}(M)[7,9]$.

Theorem 3.2. The $\Lambda$-minimal $\Lambda$-extension of a generalised Seifert fibration $\mathcal{F}$ on a connected, compact, simply-connected manifold $M$ coincides with that of the fibration $\mathcal{L} \longrightarrow M \longrightarrow B \Gamma$. In particular, the relative minimal model [9] is that of the universal leaf $\mathcal{L}$.

Proof: This follows because we have

$$
\Omega_{B}(\mathcal{F}) \longrightarrow \Omega(B \Gamma) \longrightarrow \Omega_{D R}(M)
$$

where the map on the left induces an isomorphism in rational cohomology. The last assertion follows from a theorem of Grivel [5]. 
Theoren 3.3. Let $\mathcal{M} \longrightarrow \mathcal{A} \longrightarrow \mathcal{B}$ be a $\Lambda$-minimal $\Lambda$-extension, where $H^{1}(B)=0$. Then, if $\mathcal{M}, \mathcal{A}$ and $B$ satisfy Poincaré duality, there is a signature product formula as follows:

$$
\operatorname{sign}(\mathcal{A})=\operatorname{sign}(\mathcal{M}) \cdot \operatorname{sign}(B)
$$

Proof: As a graded algebra, $\mathcal{A}$ is the tensor product of $\mathcal{M}$ and $\mathcal{B}$. We can put a filtration on $\mathcal{A}$ by

$$
F^{\prime \prime}(\mathcal{A})=B^{\geqslant r} \otimes \mathcal{M}
$$

Then $F$ is a descending filtration, and it is preserved by the differential. Thus, $F$ is a canonically cobounded filtration which gives rise to a convergent third quadrant spectral sequence with

$$
E_{2}=H(\mathcal{B}) \otimes H(\mathcal{M}) \Longrightarrow H(\mathcal{A}) \text {. }
$$

The signature product formula is then obtained similarly to [1], with the above spectral sequence replacing the Leray-Serre.

\section{Taut Riemannian flows}

It is well-known that a taut Riemannian flow $\mathcal{F}$ is given by a non-singular Killing vector-field $\mathcal{X}$, and if the ambient manifold $M$ is connected and compact, there is a decomposition of the de Rham algebra of $M$, up to cohomology, as follows [4]:

$$
\Omega_{D R}(M) \simeq \Omega_{B}(\mathcal{F}) \otimes \Lambda X
$$

where $\Lambda X$ denotes the exterior algebra with one generator of degree one with the trivial differential. This describes the minimal model of $\mathcal{F}$. Thus, it follows from Theorem 3.3 that the signature of $M$ is zero.

\section{REFERENCES}

[1] S.S. Chern, F. Hirzebruch and J.P. Serre, 'On the index of a fibered manifold', Proc. Amer. Math. Soc. 8 (1957), 587-596.

[2] D. Epstein, 'Foliations with all leaves compact', Ann. Inst. Fourier, Grenoble 26 (1976), 265-282.

[3] E. Ghys, 'Feuilletages riemanniens sur les variétés simplement connexes', Ann. Inst. Fourier, Grenoble 34.4 (1984), 203-223.

[4] W. Greub, S. Halperin and R. Vanstone, Connections, curvature and cohomology Vol 2 (Academic Press, New York, 1973).

[5] P. Grivel, 'Formes différentielles et suites spectrales', Ann. Inst. Fourier, Grenoble 28.3 (1979), 17-37. 
[6] A. Haefliger, 'Groupoïdes d'holonomie et classifiants', Astérisque 116 (1984), $70-97$.

[7] S. Halperin, 'Lectures on minimal models', Publ. Internes de l'UER de Math Pures et Appl., Univ. de Lille I 111 (1977).

[8] F. Kamber and P. Tondeur, 'Foliations and metrics', Proc. Special Year in Geometry, Maryland (1981-82), in Prog. in Math. 32 (Birkhäuser, Boston, 1983), pp. 103-152.

[8] D. Lehmann, 'Modèle minimal relatif des feuilletages', in Lect. Notes in Math. 1183 (Springer, Berlin, Heidelberg, New York, 1986), pp. 250-258.

Department of Mathematics

National University of Singapore

Kent Ridge 0511

Republic of Singapore 